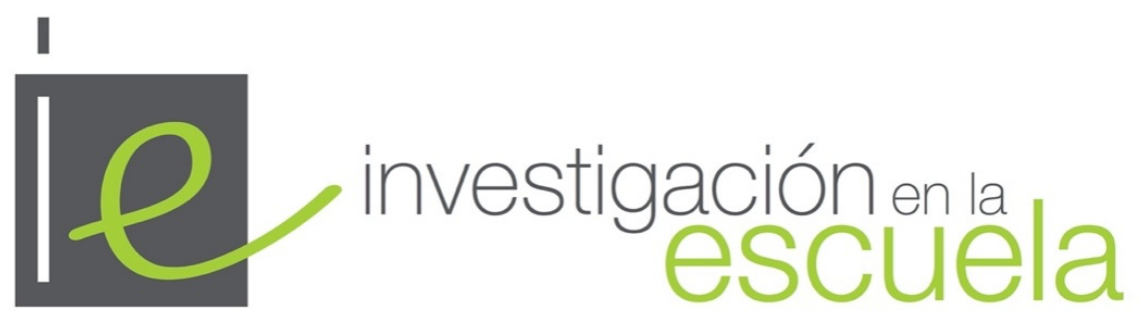

Revista de Investigación e Innovación Educativa nº 103, 2021 | e-ISSN 2443-9991

\title{
El huerto escolar desde un enfoque indagativo: investigando las lombrices ${ }^{1}$ \\ The school garden from an Inquiry-Based Learning approach: studying worms
}

iD José Luis Carballido Morejón es Maestro de Educación Primaria en el CEIP Príncipe de Asturias, Sevilla (España) · joseluis.carballido@pdasturias.es·https://orcid.org/0000-0002-6637-131X

iD Dra. Hortensia Morón Monge es Profesora Ayudante Doctor en la Facultad de Ciencias de la Educación Universidad de Sevilla (España)·hmoron@us.es ·https://orcid.org/0000-0002-3298-5911

iD Dra. Ma Paula Daza Navarro es Profesora Catedrática de Escuela Universitaria en la Facultad de Ciencias de la Educación Universidad de Sevilla (España)·pdaza@us.es·http://orcid.org/0000-0001-5170-1868

Cómo citar este artículo

Carballido Morejón, J. L, Morón-Monge, H., y Daza Navarro, Ma P. (2021). El huerto escolar desde un enfoque indagativo: investigando las lombrices. Investigación en la Escuela, 103, 75-93. doi: http://dx.doi.org/10.12795/IE.2021.i103.06

Resumen. El presente trabajo es una investigación basada en una experiencia educativa realizada con 21 estudiantes de quinto de primaria en un colegio de la provincia de Sevilla (España), surgida a partir de la observación de las lombrices en el huerto escolar. A raíz de esta situación se desarrolla dicha experiencia desde un enfoque metodológico indagativo o IBL de carácter abierto. Se expone, a modo de resultados, las oportunidades didácticas de este recurso para la enseñanza de la ciencia, así como su impacto formativo en el alumnado. Para la recogida y análisis de datos se ha diseñado una parrilla de observación compuesta por cinco categorías (habilidades científicas, actitudes positivas hacia la ciencia, comportamiento y uso respetuoso de materiales e instrumentos, hábitos saludables y sensibilización medioambiental) basadas en las recomendaciones del currículum de educación primaria de Andalucía y la literatura de revisión. En los resultados se contrasta cualitativamente la situación de partida (pre-experiencia) con los aprendizajes alcanzados al final de la experiencia (post-experiencia) donde comprobamos cómo han mejorado en las categorías observadas. Finalmente, este trabajo pone en valor el uso de este recurso desde un enfoque interdisciplinar de la enseñanza para mejorar no solo conocimientos y habilidades científicas, sino también para fomentar hábitos de respeto y sensibilización medioambiental.

Abstract. The present work is an investigation based on an education experience carried out with 21 students in a school of Seville (Spain). As a result of the observation of some worms in the school garden, this experience is developed from an Inquiry-Based Learning approach. The educational opportunities of this resource for the teaching of science and its formative impact on students are exposed as results. For the collection and analysis of data, an observation checklist has been designed composed of five categories (scientific skills, positive attitudes towards science, behaviour and respectful use of materials and instruments, healthy habits and environmental awareness) based on the recommendations of the curriculum of Andalusian and the literature review. In the results, the initial situation (preexperience) is qualitatively contrasted with the learning achieved at the end of the experience (post-experience) where we check how they have improved in the observed categories. Finally, this work highlights the use of this resource from an interdisciplinary approach to teaching to improve not only scientific knowledge and skills, but also to promote habits of respect and environmental awareness.

${ }^{1}$ Se dispone de la autorización expresa de las familias para la realización y publicación de las fotografías de los menores (figuras 2 4) para fines educativos y divulgativos. 
Palabras clave $\cdot$ Keywords

Enseñanza de las ciencias, enseñanza primaria, huerto escolar, enfoque interdisciplinario, sensibilización ambiental. Science education, primary education, school garden, learn in context, interdisciplinary approach, environmental awareness.

\section{Introducción}

La población mundial se encuentra en un momento de incertidumbre como consecuencia de la actual crisis sanitaria por la COVID-19. Igualmente, el momento de emergencia climática declarada en el 2019, la pérdida de biodiversidad de especies, la acumulación de microplásticos en la cadena alimentaria en los mares y océanos, son solo algunos ejemplos del desequilibrio medioambiental y los problemas socioambientales a los que nuestro mundo hoy se enfrenta (Morón-Monge et al., 2020).

La educación no se mantiene al margen de estas cuestiones, así lo recogen distintos informes educativos e instituciones internacionales (National Research Council, 2001) o el informe Rocard (Rocard et al., 2007) y nacionales -informe ENCIENDE- (COSCE, 2011) que recomiendan una enseñanza de las ciencias real, útil para el día a día de las personas. Igualmente, el currículum educativo español, establecido por la Ley Orgánica 8/2013, de 9 de diciembre, para la mejora de la calidad educativa (LOMCE) y, particularmente, en la Orden del 17 de marzo del 2015, por la que se desarrolla el currículo correspondiente a la Educación Primaria en Andalucía, recogen esta perspectiva de la enseñanza de las ciencias a partir de la promoción de distintos escenarios de aprendizaje para alcanzar un aprendizaje contextualizado (Franco Mariscal et al., 2017; Morón-Monge et al., 2020). En dichos documentos educativos, se conceden especial relevancia a los espacios naturales y socioculturales como escenarios de aprendizajes. En el área de Ciencias Naturales, se proponen explícitamente las actividades al aire libre como recurso educativo (huertas escolares, jardines botánicos, cuidados de animales, realización de itinerarios, etc.), y en el caso de las Ciencias Sociales, las visitas a empresas, fábricas, zonas protegidas, monumentos, etc.

De la misma manera, lo expresan distintos estudios (Aguilera, 2018; Morales et al., 2014; Zamalloa et al., 2014) sobre la contribución de las salidas de campo a la construcción del conocimiento científico y al desarrollo de estrategias científicas. Las salidas fuera del aula recogidas a nivel internacional como outdoor education u outdoor learning, son entendidas como toda experiencia fuera del aula, en el medio natural o urbano, incluyéndose igualmente aquellas realizadas en los huertos escolares (Dillon et al., 2006; Gelter, 2000; Harris, 1999; Waite, 2011). Este tipo de experiencias, contribuyen a un aprendizaje experiencial y significativo (Tal \& Morag, 2009), sensibilizan al alumnado sobre el espacio socio natural, fomentan actitudes de respeto y cuidados sostenibles (Amórtegui et al., 2017; Costillo et al., 2014; Zamolloa et al., 2014; Morón-Monge et al., 2020), entre otras de sus múltiples bondades educativas.

Desde esta perspectiva defendemos una enseñanza de las ciencias centrada en la resolución de problemas cotidianos y contextualizada a la realidad para alcanzar una alfabetización científica (Sadler, 2004; Zeidler et al., 2005). Para hacer frente a estas cuestiones, es necesario su comprensión, tomar decisiones y actuar, desde un planteamiento del currículum basado en competencias y desde el activismo ciudadano (Contreras, 2016; Hodson, 2014; Pedrinaci et al., 2012).

En este sentido, el huerto escolar como outdoor learning puede ser un buen recurso educativo que nos permita alcanzar esta finalidad de la enseñanza de las ciencias. El huerto escolar como recurso no es una temática novedosa, así nos lo indica la literatura de revisión donde se observa un interés creciente, tanto en el panorama nacional (Ceballos, 2017; Eugenio-Gozalvo et al., 2019; Eugenio-Gozalbo, et al., 2020; Vílchez y Escobar, 2014; Nicolás et al., 2014; Rodríguez-Marín, et al., 2015; Ruiz-Gallardo et al., 2013; Sáez Bondía et al., 2017; Zambrano-Quintero et al., 2018) como internacional (Dring et al., 2020; Klemmer et al., 2005; Morgan et al., 2010; Ratcliffe et al., 2011; Robinson \& Zajicek, 2005).

Centrándonos en nuestro estudio enmarcado en la provincia de Sevilla, se observa que a pesar de ser este recurso cada vez más habitual en las escuelas y usado en las distintas etapas educativas (infantil hasta incluso bachillerato), su práctica educativa es muy diversa y con un distinto rendimiento pedagógico. Así nos lo señalan los estudios de Vílchez et al. (2012) y Vílchez y Escobar (2014) en la provincia de Sevilla y más recientemente el de Ceballos (2017). Dichos trabajos muestran como efectivamente cada vez más centros de esta provincia adoptan los huertos en sus prácticas educativas, sin embargo, esto no implica que su rendimiento didáctico sea el más deseable. De hecho, la gran mayoría usan los huertos escolares desde una perspectiva didáctica tradicional, centrado en hacer actividades desvinculadas de la programación curricular, de carácter meramente lúdico, como ampliación de contenidos teóricos y donde el docente tiene el mayor protagonismo (Ceballos, 2017; Vílchez y Escobar, 2014). Por el contrario, son pocos los centros 
de esta región que lleven a cabo experiencias educativas indagatorias a partir del huerto escolar (Ceballos, 2017) como la que este trabajo aquí recoge.

\subsection{E1 huerto escolar desde una perspectiva indagatoria}

El huerto escolar a diferencia de otros recursos educativos, con una intencionalidad didáctica prestablecida, puede permitirnos trabajar diferentes áreas de conocimiento, desde distintas perspectivas didácticas. Como señalan Fernández-Arroyo et al. (2013) el huerto como recurso didáctico, se puede utilizar desde muchos enfoques educativos, lo que supone un planteamiento flexible y abierto de los objetivos, en el que cada docente puede concederle distintos usos. En este sentido, el término affordance, definido por Gibson (1979) sirve para describir lo que un espacio fuera del aula puede ofrecer o invita, es decir sus posibilidades educativas y funcionales (Sanz et al., 2021). De la misma manera se traslada este concepto a la competencia científica (scientific affordance) como aquellos espacios fuera del aula que pueden ofrecer oportunidades para la enseñanza de las ciencias (Sanz et al., 2021).

Esta versatilidad de huerto escolar como recurso educativo tendrá distinto impacto educativo en función de la perspectiva educativa que deseemos promover. Nos decantamos por la perspectiva integradora de la educación ambiental (Rodríguez-Marín et al., 2015) para este trabajo, centrada en los niños/as como potencial agente para el cambio socioambiental y con un enfoque metodológico indagativo. Dicha perspectiva permite tratar aspectos teóricos, prácticos, lúdicos desde los procesos de enseñanza-aprendizaje para el tratamiento de los problemas socioambientales y el decrecimiento (Rodríguez-Marín et al., 2015). En la misma línea, Zambrano-Quintero et al. (2018) defienden el huerto escolar como escenario para el desarrollo de competencias críticas e investigativas, que favorezcan la adquisición de nuevos saberes. Estos autores conciben el huerto escolar, como estrategia que dinamiza el proceso de aprendizaje, a partir de una enseñanza activa, dinámica, trabajando desde un entorno vivo y cambiante en el que se pueden desarrollar actividades educativas que además benefician a la institución, familia y comunidad en general (ZambranoQuintero et al., 2018).

En este sentido, los métodos de enseñanza basados en la indagación o el aprendizaje basado en la indagación (Inquiry-Based Learning approach, IBL) es uno de los temas más debatidos en didácticas de las ciencias, aunque su conceptualización suele generar confusión (Ferrés et al., 2014). Como estas autoras señalan podemos entender la indagación como objeto de aprendizaje (aprender hacer ciencia y aprender sobre ciencia) o como modelo didáctico (aprender ciencia por medio de la indagación). Durante las actividades de indagación el alumnado participa de forma activa en la comprensión de ideas científicas actuando el docente como guía, además de entender la forma en la que los científicos estudian los fenómenos naturales (Godoy et al., 2014). El IBL mejora el interés por la ciencia en los estudiantes (Rocard et al., 2017), logra una mayor comprensión de los conceptos científicos, además de desarrollar habilidades científicas durante su procedimiento. La promoción de este tipo de experiencias contribuye al aprendizaje centrado en competencias como aconseja la OCDE (2005). En este sentido, la capacidad de indagación está relacionada con la dimensión metodológica de la competencia científica (Ferrés et al., 2014).

Concebimos el huerto escolar desde una perspectiva integradora y de indagación como modelo didáctico, orientada no exclusivamente a la formación de pequeños agricultores, ni producir alimentos, sino que compartimos el mismo enfoque de Fernández-Arroyo et al. (2013):

Simular lo que ocurre en la agricultura real para que los estudiantes puedan aprender conceptos, actitudes y procedimientos relacionados con las diferentes áreas curriculares y transversales (forma de vida y cuidado de las plantas, qué son las labores agrícolas y que destrezas hay que utilizar, cómo observar y cómo clasificar, qué es un ecosistema, qué recursos obtenemos del campo, cómo medir lo que crece una planta, por qué y cómo debemos cuidar el medio, desarrollo de hábitos de consumo saludables, etc. (p. 704).

\subsection{Algunas oportunidades educativas del huerto escolar desde nuestro centro: antecedentes}

Algunas de las oportunidades didácticas que ofrece el huerto escolar y que ejemplifican el uso didáctico de este recurso en nuestro centro educativo, ya han sido recogidas dentro del video polimedia: ¿Cómo trabajar la ciencia en el huerto escolar? (2020). En este video polimedia, se describe de forma sintética cómo el aprendizaje no solo surge en las aulas, sino en cualquier contexto, siendo el huerto escolar un escenario perfecto para tratar distintas áreas de conocimiento de forma integrada. Por lo que esta experiencia se basa en estos antecedentes previos y forma parte del proyecto "Del planeta al buerto" en el que participa nuestro CEIP. 
Dicho proyecto comenzado en el curso escolar 2018-2019, tiene como objetivo principal, generar un contexto educativo idóneo donde favorecer la curiosidad y entusiasmo del alumnado en la adquisición de determinados conocimientos y valores, a partir de la investigación y el respeto por el medioambiente. En este sentido, la comunidad educativa de nuestro CEIP vio como una oportunidad didáctica trabajar con el huerto escolar, como instrumento inclusivo y sostenible que dotase de carácter e identidad propia al centro. Además, está apoyado por el programa escolar del Ayuntamiento de Sevilla (Huertos Urbanos de Sevilla) que ofrece formación, materiales y apoyo al profesorado durante tres horas a la semana con los distintos niveles educativos. En las tablas 1 y 2, se detalla la organización seguida y gestión del huerto por la escuela.

Como se observa en la tabla 1, en este proyecto además de intervenir gran parte de la comunidad educativa, también participan familias, normalmente tres por aula, además de personal especialista como son los hortelanos y hortelanas que proporcionaba la asociación.

Tabla 1

Sectores implicados en la participación del proyecto "Del planeta al buerto"

\begin{tabular}{lll}
\hline $\mathbf{N}^{\mathbf{o}}$ de Docentes & Familias Implicadas & $\mathbf{N}^{\mathbf{o}}$ de Hortelanos/as (Asociación) \\
\hline 17 & $3 /$ aula & $5-6$ \\
\hline
\end{tabular}

En la tabla 2, se recoge el cronograma de trabajo en el huerto por niveles educativos, desde Educación Infantil hasta Primaria. Igualmente, a veces también participan antiguos alumnos del centro, ayudando a las tareas de mantenimiento, diseño y divulgación de los resultados del proyecto.

Tabla 2

Uso del huerto por nivel educativo: reparto temporal

\begin{tabular}{|c|c|c|c|c|}
\hline \multirow[t]{2}{*}{ Nivel educativo } & \multicolumn{2}{|c|}{ Frecuencia } & \multirow[t]{2}{*}{ Día } & \multirow[t]{2}{*}{ Tiempo } \\
\hline & Semanal & Mensual & & \\
\hline $\begin{array}{l}\text { INFANTIL } 4 \\
\text { AÑOS }\end{array}$ & $\mathrm{X}$ & & Martes & \multirow{7}{*}{45 minutos/sesión } \\
\hline PRIMERO & $\mathrm{X}$ & & Martes & \\
\hline SEGUNDO & $\mathrm{X}$ & & Lunes & \\
\hline TERCERO & $\mathrm{X}$ & & Martes & \\
\hline CUARTO & & $\mathrm{X}$ & Lunes / martes & \\
\hline QUINTO & $\mathrm{X}$ & & Lunes / martes & \\
\hline SEXTO & $\mathrm{X}$ & & Lunes & \\
\hline
\end{tabular}

En los cuatro años que llevamos usando el huerto como recurso educativo este se ha convertido en un recurso muy útil para la enseñanza, realizándose distintas actividades para desarrollar contenidos académicos a partir de pequeñas investigaciones. Para su desarrollo, intervienen además de los docentes y alumnado, los familiares y otros agentes públicos y privados (hortelanos/as, expertos/as en el tema de la agricultura, etc.).

Desde el área de la enseñanza de las ciencias, el huerto escolar, tiene como principal fin: crear hábitos de respeto hacia el medio ambiente mediante actividades de sensibilización. Para ello, se han ido creando rutinas diarias de trabajo que fomenten un aprendizaje significativo, a partir del desarrollo de habilidades científicas (observación, inferencia, recogida de datos y análisis, argumentación, etc.) y concienciación de los problemas socioambientales. Particularmente, algunos de los conocimientos disciplinares que pueden ser trabajados a partir del uso del huerto escolar como recurso han sido recogidos en la tabla 3. Estas son algunas de las oportunidades didácticas o "affordance" que nos puede ofrecer el huerto escolar, aunque en este trabajo nos centramos en exponer únicamente aquellas derivadas de experiencia educativa a partir del estudio de las lombrices. Aunque en dicha tabla hayamos separado las distintas áreas de conocimiento por filas, el huerto permite trabajar de forma interdisciplinar a partir de su cuidado y mantenimiento. Por lo que esta tabla solo recoge algunas de las múltiples posibilidades educativas que puede ofrecer los huertos escolares. La elección de una u otra va a depender de los objetivos y finalidades didácticas propuestas y, su impacto educativo de la perspectiva didáctica empleada (Carballido, 2020). 
Tabla 3

Algunas oportunidades del Huerto escolar

\begin{tabular}{|c|c|c|}
\hline $\begin{array}{l}\text { Área de } \\
\text { conocimiento }\end{array}$ & $\begin{array}{l}\text { Contenidos } \\
\text { y } \\
\text { Habilidades }\end{array}$ & Ejemplos: actividades y conocimientos específicos \\
\hline \multirow{5}{*}{ Lengua } & Vocabulario & $\begin{array}{l}\text { Vocabulario específico del huerto (herramientas del huerto, tipos de } \\
\text { hortalizas y verduras, técnicas de sembrado...) }\end{array}$ \\
\hline & $\begin{array}{l}\text { Expresión } \\
\text { oral }\end{array}$ & $\begin{array}{l}\text { Exposiciones en diversos ámbitos (feria de la ciencia, centro cívico, } \\
\text { otros compañeros/as...) }\end{array}$ \\
\hline & \multirow{3}{*}{$\begin{array}{l}\text { Expresión } \\
\text { escrita }\end{array}$} & Redacción de procesos (crecimiento de una planta) \\
\hline & & Texto instructivo (redactar cómo debe hacerse una siembra) \\
\hline & & $\begin{array}{l}\text { Realizar cartas (dirigirse a través de un email a un experto en la } \\
\text { materia o investigación para solicitar ayuda) }\end{array}$ \\
\hline \multirow{5}{*}{ Matemáticas } & \multirow[b]{3}{*}{$\begin{array}{l}\text { Unidades de } \\
\text { medida }\end{array}$} & Dinero (precios de los materiales que se emplean) \\
\hline & & El tiempo (calendario, semanas, días...) \\
\hline & & $\begin{array}{l}\text { Unidades de masa (peso de las hortalizas, unidades necesarias para una } \\
\text { receta, cantidad de productos para echar en una compostera para la } \\
\text { fabricación de humus...) }\end{array}$ \\
\hline & Números & $\begin{array}{l}\text { Resolución de situaciones problema (Mediciones de bancales, \% de } \\
\text { semilleros, fracciones del huerto sembradas...) }\end{array}$ \\
\hline & $\begin{array}{l}\text { Estadística y } \\
\text { probabilidad }\end{array}$ & Gráficos (hortalizas de temporada) \\
\hline \multirow{7}{*}{$\begin{array}{l}\text { Ciencias } \\
\text { Naturales }\end{array}$} & \multirow{3}{*}{$\begin{array}{l}\text { Los seres } \\
\text { vivos }\end{array}$} & Clasificación de seres vivos según sus características \\
\hline & & Ecosistemas: el huerto como ecosistema \\
\hline & & Reciclaje: el compost en el huerto \\
\hline & $\begin{array}{l}\text { El ser } \\
\text { humano y la } \\
\text { salud }\end{array}$ & La alimentación sana: "real food" y alimentos de temporada \\
\hline & $\begin{array}{l}\text { El suelo y su } \\
\text { formación }\end{array}$ & $\begin{array}{l}\text { Tipos de suelo, composición química, mantenimiento para la fertilidad } \\
\text { agrícola. }\end{array}$ \\
\hline & La materia y & La materia: el ciclo de la materia a partir del huerto \\
\hline & la energía & La energía: usos, movimiento y transformación \\
\hline \multirow[t]{3}{*}{$\begin{array}{l}\text { Ciencias } \\
\text { Sociales }\end{array}$} & Historia & $\begin{array}{l}\text { Importancia de la agricultura en la historia y el rol de la mujer en la } \\
\text { agricultura. } \\
\text { Técnicas de cultivo tradicionales y actuales (barbecho, invernaderos, } \\
\text { hidropónicos, los transgénicos, etc.) } \\
\text { Herramientas e instrumentos de siembra. } \\
\text { Origen de las hortalizas (Importancia del comercio con América). }\end{array}$ \\
\hline & $\begin{array}{l}\text { El clima y el } \\
\text { relieve }\end{array}$ & $\begin{array}{l}\text { Las estaciones del año y el sembrado: calendario de siembra. } \\
\text { El clima y el relieve: para la siembra y las distintas variedades. }\end{array}$ \\
\hline & Economía & Autoproducción, la economía circular, el ciclo del producto, etc. \\
\hline \multirow[b]{2}{*}{$\begin{array}{l}\text { Educación } \\
\text { Artística }\end{array}$} & \multirow{2}{*}{$\begin{array}{l}\text { Expresión } \\
\text { artística y } \\
\text { creatividad }\end{array}$} & $\begin{array}{l}\text { Canciones, dibujos, carteles, cómics sobre el huerto, sus plantas y las } \\
\text { estaciones del año. }\end{array}$ \\
\hline & & $\begin{array}{l}\text { Manualidades con materiales reutilizados y/o del propio huerto. } \\
\text { Actividades de reciclaje. }\end{array}$ \\
\hline $\begin{array}{l}\text { Lengua } \\
\text { Extranjera }\end{array}$ & Vocabulario & Vocabulario en inglés y francés propio del huerto escolar. \\
\hline
\end{tabular}

Teniendo presente este panorama, nuestro objetivo principal es presentar una experiencia educativa en marcada en el proyecto anteriormente descrito con el alumnado de quinto de primaria donde se usa el huerto el escolar como recurso educativo para la alfabetización científica desde un enfoque indagativo. A raíz de la observación de lombrices en la tierra del huerto escolar y del interés que suscita en el alumnado dicho "descubrimiento", se desarrolla todo un proceso de investigación. Con la finalidad última de mostrar algunas de sus implicaciones educativas en el alumnado, haciendo especial énfasis en las habilidades científicas y la mejora del comportamiento de los estudiantes, interés y motivación hacia las ciencias. Señalar, que el interés 
de este trabajo es doble pues a pesar de existir una extensa bibliografía sobre esta temática, son pocas las prácticas educativas como la que aquí se exponen de la mano de docentes de primaria en ejercicio. Igualmente, como formadores de docentes pretendemos también que sirva esta experiencia de ejemplo a otros maestros/as en formación y en ejercicio que deseen trabajar con el huerto escolar aprovechando sus distintas oportunidades educativas.

\section{Material y métodos}

\subsection{Metodología}

El presente estudio tiene un enfoque cualitativo, que busca comprender e interpretar la realidad de los fenómenos que se estudian dentro de su contexto natural o in situ (Bisquerra, 2009; Zambrano-Quintero et al., 2018). El alcance de la investigación es descriptiva-interpretativa, ya que se basa en la observación y recogida de datos, desde una evaluación continua y formativa (Bisquerra, 2009). Desde esta perspectiva se diseña el instrumento de recogida de datos, una hoja de registro (tabla 4), y se analiza (Bisquerra, 2009; Denzin \& Lincoln, 2012; Abril-López et al., 2021), siendo este instrumento tanto de recogida como de análisis cualitativo a partir de la interpretación de los datos que se hacen por cada categoría establecida.

El concepto de observación depende del tiempo y el contexto en el que se aplique. Desde nuestro contexto, la observación de los procesos enseñanza-aprendizaje en el ámbito escolar, lo entendemos como una técnica de investigación educativa, de carácter intencionado, específico y sistemático que requiere de una planificación previa para la recogida de información del problema o cuestión que nos preocupa o interesa (Rekalde et al., 2014). Como proceso de recogida de información, la observación es fundamental para la evaluación formativa y así conseguir mejorar los procesos de enseñanza y aprendizaje (Fuertes Camacho, 2011). En este trabajo se lleva a cabo una observación directa, participativa (se realiza en el contexto), de campo y estructurada.

Esta observación realizada por el docente forma parte de su diario pedagógico, entendiendo este último como un sistema de registros usado para señalar las situaciones más notables acontecidas a lo largo de los procesos de enseñanza-aprendizaje, tanto en lo disciplinar como en lo práctico (Porlán y Martín, 2000). El carácter secuencial del diario nos permite identificar situaciones y/o acciones que se repiten y, por tanto, sirven como fuente para realizar exploraciones sistemáticas de la labor educativa realizada por el docente. El análisis del diario, la clasificación de datos, codificación y categorización de los datos de las distintas sesiones observadas puede convertirse en un modelo de evaluación progresiva de las competencias (Monsalves y Pérez, 2012).

Desde este enfoque, se lleva a cabo la observación en este estudio, realizándose a lo largo de toda la experiencia educativa (aproximadamente un trimestre escolar). Señalar que a estos tres meses se le debe sumar el periodo previo a la experiencia (un mes aproximado) para que el alumnado se familiarice con el huerto escolar y el periodo posterior a la experiencia, de seguimiento de las costumbres y rutinas escolares trabajadas. Por lo que la observación aquí realizada, tiene como finalidad recoger información acerca de los comportamientos y actitudes del alumnado relativos a la ciencia, así como las habilidades científicas alcanzadas durante el proceso de enseñanza-aprendizaje al igual como realizan otros estudios (Fuertes Camacho, 2011). Otros trabajos como el de Maldonado et al. (2018) y Eugenio-Gozalvo et al. (2019), usan también el diario como fuente de investigación del docente en el huerto escolar. No usamos esta técnica para la evaluación de contenidos de carácter conceptual no siendo además objeto de este trabajo en particular. Por el contrario, nos centramos en analizar cinco categorías observacionales relacionadas con las actitudes, procedimientos y comportamientos del alumnado (tabla 4), configurándose así nuestro instrumento de recogida de datos (hoja de registro). La selección de las categorías observacionales se ha hecho atendiendo a las recomendaciones del currículum educativo de Andalucía de la etapa de educación primaria en el área de las ciencias. Además, los distintos descriptores se han detallado y complementado con la literatura de revisión.

El procedimiento de recogida de datos y análisis se ha llevado a cabo a partir de esta hoja de registro, donde se van anotando por categorías observables, sí se cumplen o no por los estudiantes y en qué momento o actividad se lleva a cabo la observación. La entrada "otras observaciones", sirve para detallar y graduar aspectos relevantes de la observación que se hayan recogido tanto como sí o no, de tal forma que nos permiten determinar en qué medida se observa el cumplimiento de dichos descriptores. Esta hoja de registro forma parte del diario del docente, siendo completada en cada sesión para cada grupo de trabajo (cinco grupos de alumnos) durante todo el proceso que duró la experiencia. 
Tabla 4

Hoja de registro del docente

\begin{tabular}{|c|c|c|c|c|}
\hline $\begin{array}{l}\text { Categorías } \\
\text { Observables }\end{array}$ & Descriptores & Sí/No & $\begin{array}{l}\text { Momento o } \\
\text { Actividad }\end{array}$ & $\begin{array}{l}\text { Otras } \\
\text { observaciones }\end{array}$ \\
\hline $\begin{array}{l}\text { I. Habilidades } \\
\text { científicas }\end{array}$ & $\begin{array}{l}\text { Observación, rigor ante la precisión en } \\
\text { la recogida de datos, métodos y } \\
\text { procedimientos de trabajo, la } \\
\text { utilización de instrumentos de medida, } \\
\text { interpretación de la información, } \\
\text { representación de datos y } \\
\text { contrastación, argumentación de los } \\
\text { resultados. }\end{array}$ & & & \\
\hline $\begin{array}{l}\text { II. Actitudes } \\
\text { positivas hacia la } \\
\text { ciencia }\end{array}$ & $\begin{array}{l}\text { Construcción del pensamiento basado } \\
\text { en la curiosidad, interés, creatividad, } \\
\text { apertura a nuevas posibilidades, ideas, } \\
\text { experimentaciones. Visión positiva de } \\
\text { las personas que hacen ciencia, } \\
\text { superación de visiones estereotipadas } \\
\text { de las ciencias, supersticiones y } \\
\text { prejuicios, búsqueda de distintas } \\
\text { fuentes de información y } \\
\text { contrastación. }\end{array}$ & & & \\
\hline $\begin{array}{l}\text { III. Grado de } \\
\text { sensibilización } \\
\text { medioambiental }\end{array}$ & $\begin{array}{l}\text { Actitud responsable ante los } \\
\text { problemas asociados a la intervención } \\
\text { del ser humano en la naturaleza, el uso } \\
\text { de los recursos naturales, la } \\
\text { alimentación. Entendiendo el suelo } \\
\text { como un ecosistema con vida. } \\
\text { Consumo, el cuidado de los seres } \\
\text { vivos y la igualdad de género para el } \\
\text { desarrollo de las actividades en el } \\
\text { reparto de tareas. Y entender el suelo } \\
\text { como un organismo }\end{array}$ & & & \\
\hline $\begin{array}{l}\text { IV. Grado de } \\
\text { adquisición de } \\
\text { Hábitos } \\
\text { saludables }\end{array}$ & $\begin{array}{l}\text { Hábitos de vida saludable como la } \\
\text { prevención de enfermedades, } \\
\text { trastornos alimenticios una } \\
\text { alimentación equilibrada, el cuidado } \\
\text { del propio cuerpo mediante la práctica } \\
\text { diaria de ejercicio y pensamiento } \\
\text { positivo. }\end{array}$ & & & \\
\hline $\begin{array}{l}\text { V. } \\
\text { Comportamiento } \\
\text { y uso de los } \\
\text { materiales e } \\
\text { instrumentos }\end{array}$ & $\begin{array}{l}\text { Actitudes de prevención de riesgos } \\
\text { laborales ante los instrumentos y } \\
\text { materiales de trabajo y respeto ante las } \\
\text { personas y sus ideas al compartir tareas } \\
\text { en equipos independientemente del } \\
\text { sexo o ideas sobre los mismos. } \\
\text { Seguridad en el uso de instrumentos y } \\
\text { materiales de trabajo en el huerto } \\
\text { escolar. }\end{array}$ & & & \\
\hline
\end{tabular}

En cuanto al proceso de análisis e interpretación de los datos posterior, estos han sido analizados cualitativamente donde se ha ido comparando las sesiones previas de la experiencia descritas en fases (pre: fases 0 y 1) con los datos finales de la experiencia (post: fases 3 y 4 ). 


\subsection{Contexto sociocultural de la experiencia y participantes}

El centro educativo en el que se enmarca esta experiencia didáctica está situado en la zona de las "Las Lumbreras" perteneciente al barrio de Torreblanca de los Caños, de Sevilla capital. Este es un barrio situado a las afueras de la capital hispalense y, según los datos del plan de centro, lo sitúan como una de las zonas de Sevilla más deprimidas socioeconómicamente. La tasa de ocupación laboral es muy baja, (alrededor de un $50 \%$ de paro) además de eventualidad, precariedad y baja cualificación. En términos generales, esta zona posee una población más joven al resto de la ciudad. No obstante, el nivel sociocultural es inferior al de otros barrios cercanos, solo un 20\% termina la Educación Secundaria y aproximadamente un 4\% acaban estudios universitarios, según los datos que recoge el programa educativo del CEIP. A pesar de todo lo anterior, la zona donde se desarrolla esta experiencia educativa no es la zona más deprimida, siendo la que menor nivel de fracaso escolar y absentismo presenta del barrio.

La experiencia que debajo se detalla fue llevada a cabo con 21 estudiantes (11 alumnas y 10 alumnos) de quinto curso de primaria procedentes del centro educativo descrito, con una edad comprendida entre los 10 y 12 años.

\subsection{Descripción de la experiencia educativa}

En este apartado se describe de forma detallada la experiencia didáctica realizada con los estudiantes de quinto de primaria desde el área de las ciencias naturales usando el huerto como un recurso educativo. Para ello, es necesario alinear las necesidades educativas del alumnado de este contexto socio-cultural tan determinado con las oportunidades didácticas que ofrece el huerto escolar. Para ello, en grupos de trabajo el alumnado lleva a cabo un proceso indagativo abierto para resolver un problema motivado por el docente. Decimos "motivado" porque, aunque no haya sido un problema nacido del interés del alumnado, se suscitó a partir de un problema real observado durante el mantenimiento del huerto.

De forma general, la rutina que se realiza en el huerto para su conservación es siempre la misma: observación, mantenimiento y clasificación de los seres vivos y materia inerte observada. En una de estas rutinas pueden surgir de manera dirigida o espontánea distintos problemas de interés que pueden ser abordados desde distintas disciplinas. Como, por ejemplo: las ventajas de la permacultura; la influencia de factores que intervienen en el crecimiento de las plantas; control de plagas de forma biológica y ecológica (uso de mariquitas y productos caseros elaborados por el alumnado); la fabricación de humus de lombriz y sus beneficios en las cosechas, etc. Seleccionamos esta última, fabricación de humus, para ejemplificar el uso del huerto como recurso educativo para las ciencias. Esta experiencia queda recogida en cuatro grandes fases, y una inicial, que configuran así el proceso de indagación escolar desarrollado con una duración aproximada de tres meses (febrero-abril) aunque la fase cuarta y última algunas actividades se llevaron al comienzo del tercer trimestre tal y como podemos ver en el esquema de la figura 1.

\section{Figura 1}

Sintesis de las Fases de la experiencia indagatoria

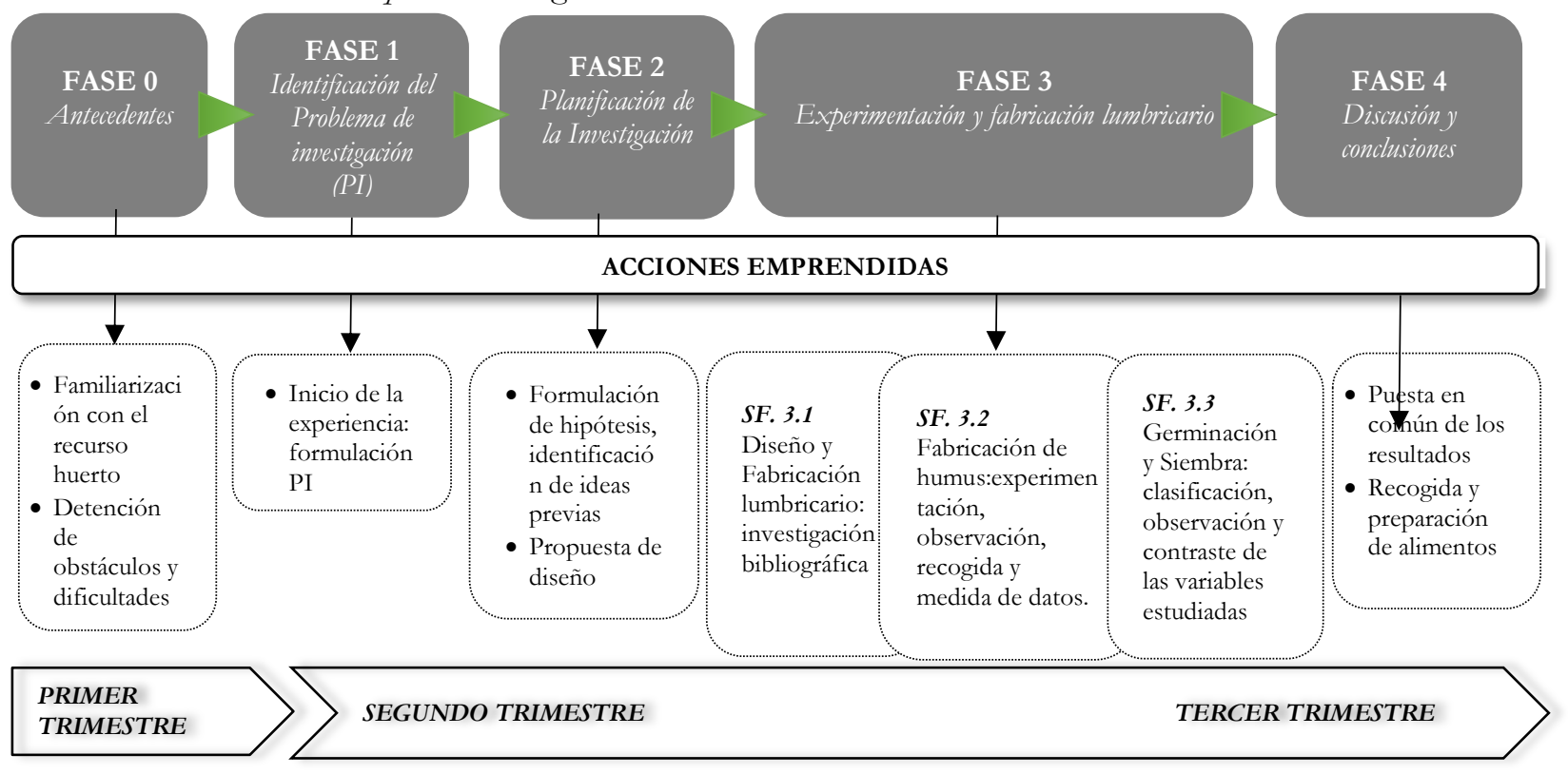


Fase 0. Antecedentes: esta fase se corresponde con el momento antes de comenzar la experiencia indagatoria durante el primer trimestre, en el que el alumnado, en grupos de trabajo, salía de forma esporádica al huerto para labores de mantenimiento rutinarias y se familiarizaba con este recurso. Por lo que dicha fase tiene como objetivo que el alumnado se familiarice con este recurso, además del docente ir observando dificultades y obstáculos en la organización y gestión de los grupos para una mayor planificación didáctica posterior.

Fase 1. Formulación del problema a investigar: al comienzo del segundo trimestre en el que el alumnado tiene una mayor integración de las rutinas de mantenimiento de la huerta, comienza esta fase. Tras una salida fuera del aula para mantener el huerto (ver figura 2), donde los estudiantes identifican, clasifican y registran los elementos vivos y materia inerte observada o cualquier otra cosa que les llame la atención; un día prestan especial atención a las lombrices encontradas en la tierra, surgiendo la siguiente cuestión: ¿Por qué están aquí estos seres vivos? Esta pregunta se trató posteriormente en el aula como se detalla en la siguiente fase.

\section{Figura 2}

\section{Estudiantes en el huerto (fase 2)}
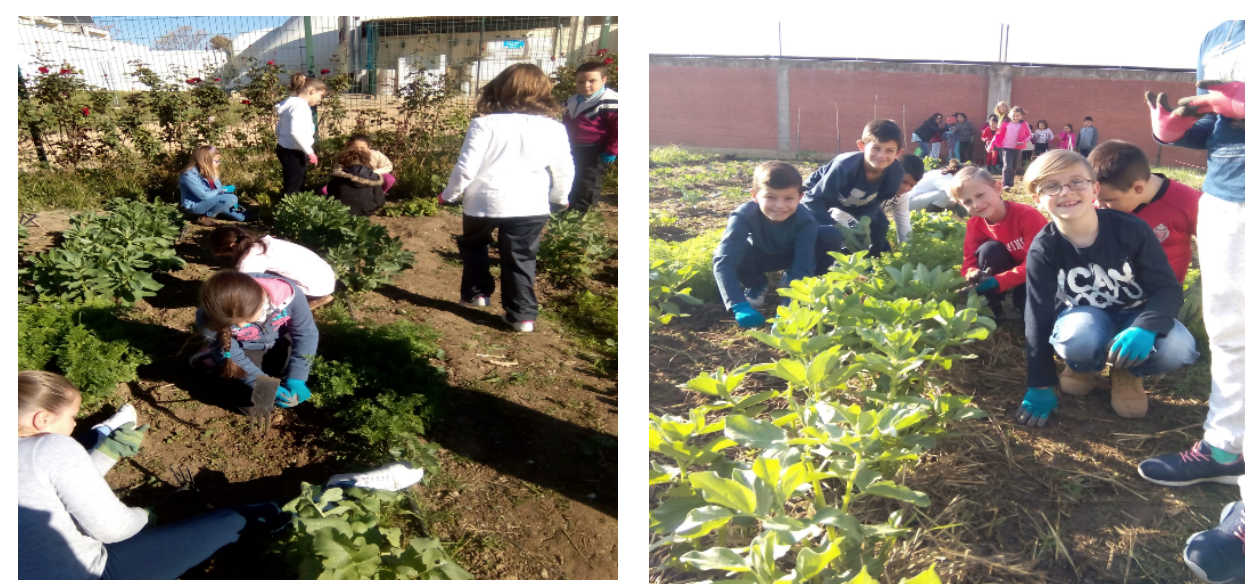

Fase 2. Planificación de la investigación: en el aula el alumnado dividido en grupos heterogéneos de trabajo (4-5 estudiantes) comienza a ofrecer distintas respuestas a la pregunta surgida. Los estudiantes formulan hipótesis, predicciones y otras posibles preguntas secundarias a raíz del debate coordinado por el docente: ¿Qué función tienen en el huerto las lombrices? ¿Qué tiene el humus de lombriz?; ¿Qué ventajas tiene?; ¿Cumple las condiciones para seguir considerando ecológico nuestro huerto? y/o ¿Qué son restos orgánicos? ¿Qué condiciones son necesarias para la conservación del lumbricario?

En esta fase se recogen las ideas previas más comunes de los estudiantes, así como sus dificultades e ideas alternativas, para ser tratadas posteriormente en el diseño de la siguiente fase. De esta puesta en común, se observa que el alumnado no entendía la relación de las lombrices con la fabricación del humus a partir de la descomposición de restos de materia orgánica y tenían dificultades para identificar los restos orgánicos de otros tipos de residuos (conocimientos sobre la composición de los materiales y el reciclado). Igualmente, se observa que los estudiantes solo reconocen el suelo como soporte físico de la vida, pero carecen de conocimientos sobre su formación, su vida microscópica y su relación con la fertilidad. Para poder observar mejor a estos seres vivos y conocer su funcionamiento y relación con la obtención del humus, se proyecta el diseño de un lumbricario. Además, de ayudarnos a responder la pregunta planteada, el diseñar un lumbricario, nos permite fabricar nuestro propio humus para el huerto.

Fase 3. Experimentación y fabricación del lumbricario: para determinar la relación de las lombrices con la mejora de las propiedades físico-químicas del sustrato, se opta por realizar una siembra sin humus de lombriz (como se realizaba siempre) y otra siembra con humus de lombriz (proporción 30/70). La variable que se estudia es humus de lombriz comparando ambas siembras, lo que nos ayudaría a determinar la influencia o no del humus con el desarrollo de las plantas. Llegando a este punto las hipótesis anteriores del alumnado fueron evolucionando y concretándose sobre la influencia del humus en la siembra. Los estudiantes consideran que las siembras con humus hacen a las plantas germinar antes, obtener mayor altura y crecer más rápido. Distinguimos Sub-Fases:

- SF.3.1. Diseño y fabricación del lumbricario: se fabricó el lumbricario con lombrices de California, en este proceso de construcción y diseño, se investigó previamente sobre cómo construirlo, qué tipo de lombrices usar, necesidades de las lombrices y principales características a partir de videos tutoriales de internet, blogs y gracias a la colaboración y ayuda de las familias y de los hortelanos de la asociación. 
- SF.3.2. Obtención del humus de lombriz: durante la fabricación del humus, el alumnado pudo observar la vida de las lombrices, su desarrollo, su relación con la cadena alimenticia como descomponedor, se estudió su anatomía, ciclo de vida, etc. Durante un mes el alumnado, por grupos, seguía una misma rutina al comenzar la clase a partir de las siguientes tareas: recogida y clasificación de restos orgánicos; anotación y registro de los cambios que se observaban en el lumbricario; y/o pesar y trocear los restos orgánicos que introducían en el lumbricario (figura 3).

Figura 3

Estudiantes fabricando el bumus de lombriz. (SF. 3.2)
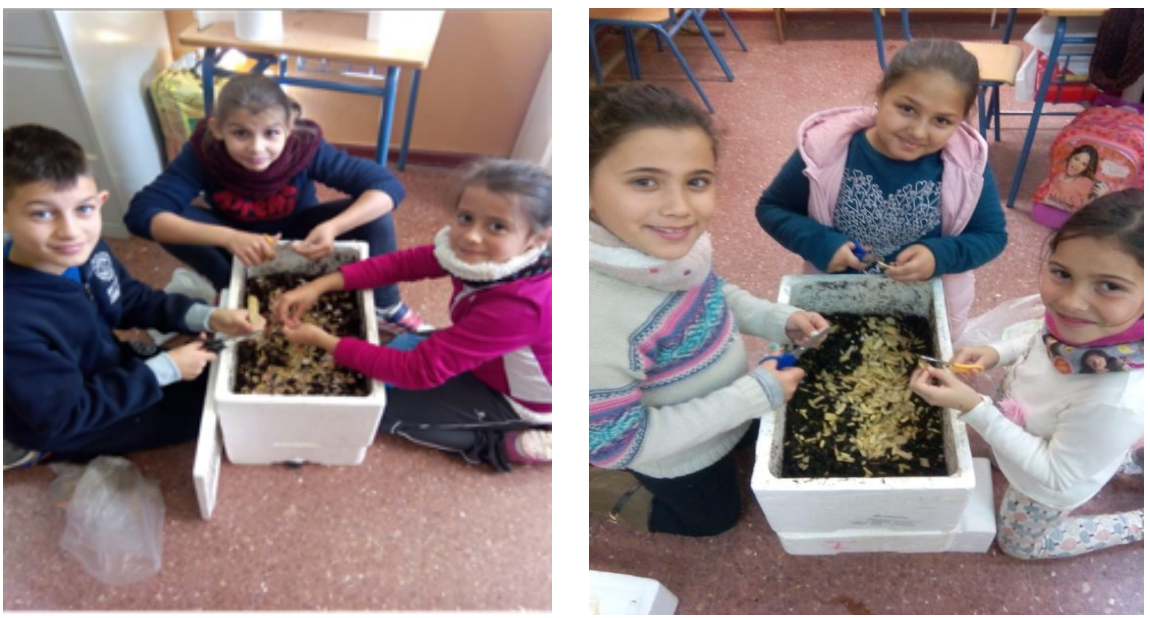

- SF.3.3. Germinación y siembra: una vez obtenido el humus de lombriz, los estudiantes divididos en grupos de trabajo realizaron distintas tareas para la preparación de las primeras siembras (figura 4): elaboración de etiquetas; clasificación y reparto de semillas (en función del tiempo de germinación, no todas germinan a la vez: 5-15 días); preparación de la tierra de cultivo (calculando el porcentaje idóneo de humus de lombriz y mantillo, haciendo proporciones/fracciones -entre un 30\% de humus y $70 \%$ de mantillo); fabricación de pequeños invernaderos, etc. Se usaron semillas de berenjenas, tomates, pimientos, calabacines y pepinos, siguiendo el calendario de siembra.

Figura 4

Estudiantes preparando los semilleros (SF. 3.3)

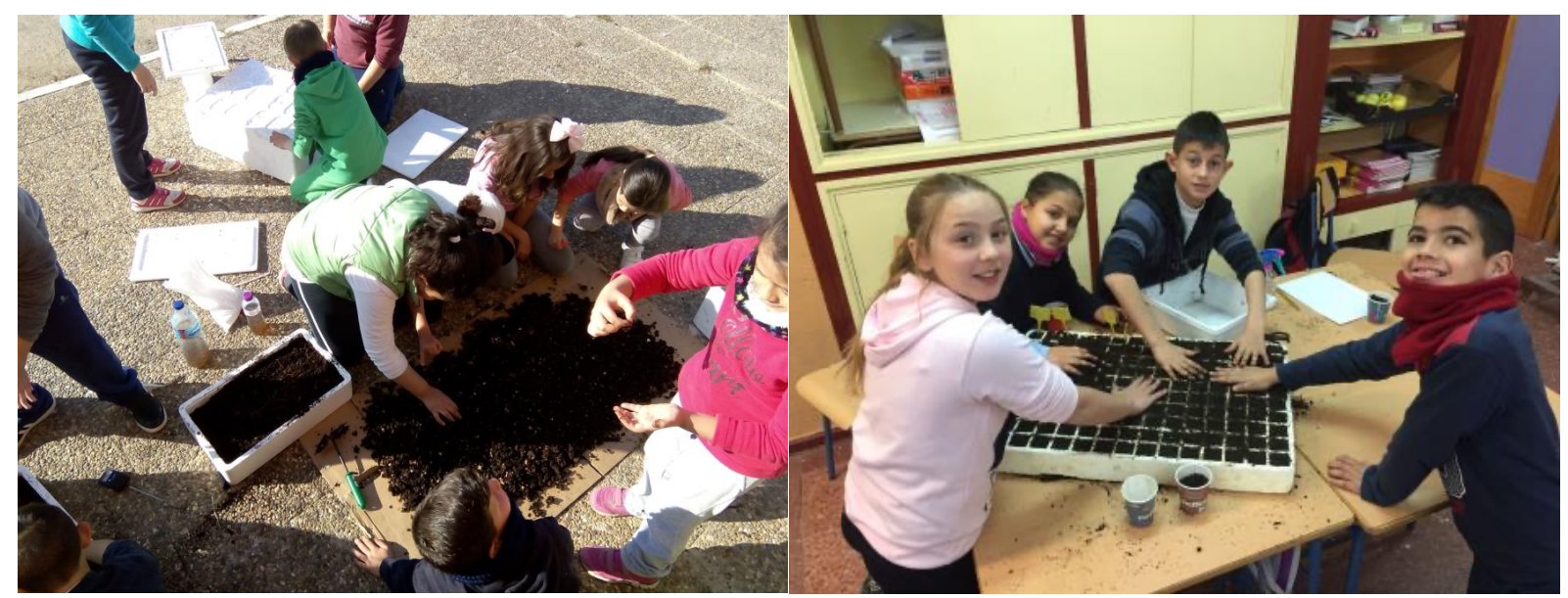

Además, en esta sub-fase 3.3 surgió otra cuestión relacionada con la siembra: la posible influencia del tipo de semillero en la fase de germinación. El alumnado no tenía claro si el tipo de semillero era una variable a tener en cuenta, por lo que se controló esta nueva variable mediante el uso de distintos tipos de semilleros (individuales, en caja y vaso) y cómo afectaba a la germinación de las semillas. Para ello, se estuvo realizando una observación del proceso de germinación y evolución de los plantones a partir de una hoja de registro (tabla 5) que diferenciaba los distintos tipos de semilleros para cada una de las dos siembras (con humus/sin humus). En estas hojas se iban anotando los días y los centímetros que crecían las plantas para después 
hacer una gráfica de crecimiento. Para ello, se anotaba diariamente el número de semillas que germinaban cuando asomaban fuera de la tierra. Después se anotaban las tres o cuatro plántulas que más medían de cada semillero. En las observaciones se podían registrar otros aspectos relevantes sobre: la tierra (si estaba seca o húmeda) o el estado de las plantas (si se estropeaba alguna, y la tenían que quitar), etc.

Tabla 5

Hoja de registro del estudiante: germinación de las semillas

\begin{tabular}{lllll}
\hline Día & $\begin{array}{l}\text { Germinan } \\
\left(\mathbf{N}^{\circ}\right)\end{array}$ & Miden $(\mathrm{cm})$ & Riego & Observaciones \\
\hline Hortaliza & & & \\
\hline Caja & & \\
\hline Individual & & \\
\hline Vaso & & \\
\hline
\end{tabular}

Fase 4. Discusión de los resultados y conclusión: Con los datos recogidos en las distintas hojas de registro del estudiante (tabla 5) y con todo el proceso anterior realizado, se obtuvo las siguientes conclusiones. Los alumnos experimentaron cómo la siembra que estaba compuesta por humus de lombriz hacía que las plántulas crecieran antes (proceso más acelerado), pero no que fueran a ser más grandes o altas (algunas podrían serlo y otras no), además de obtener más frutos que la siembra sin humus de lombriz (esto se observó en la siembra de tomates, donde se obtuvo más frutos). En relación con la variable tipo de semillero se llegó a la conclusión que en un principio el tipo de semillero seleccionado en nuestro estudio no parecía influir en el crecimiento. Lo único que había semilleros más cómodos a la hora de trabajar con los plantones y sacarlos. Finalmente, toda esta experiencia culminó en la recogida de los productos de la huerta (pimientos, calabacines, berenjenas y algunos tomates, siendo algunos de ellos cultivados antes de comenzar la experiencia) y elaboración de comidas con la ayuda de las familias donde el alumnado degustó distintos platos con los productos obtenidos durante su experiencia educativa, ya en el tercer trimestre. Como aspecto notable, señalar que la gran mayoría de estos alumnos probaron por primera vez estas verduras, el alumnado demostraba falta de interés por estos alimentos en su dieta semanal. No obstante, durante este proceso de mantenimiento del huerto, sembrado y recogida el alumnado fue familiarizándose con estos alimentos, fomentando así su interés culinario por los mismo.

Atendiendo a la experiencia descrita, podemos observar la correspondencia de algunos de los aprendizajes desarrollados en las en las distintas fases en la tabla 6. Las Habilidades Científicas (HC) se han trabajado a lo largo de toda la experiencia, también se han desarrollado Conocimientos Científicos específicos (CC) y Actitudes Científicas (AC). Igualmente, en esta tabla se resaltan otros conocimientos trabajados desde otras áreas como las matemáticas o lengua:

- Área de las Matemáticas: durante toda la fase 3, se trabajó con las magnitudes de medida (peso de la cantidad de restos orgánicos que se le echaba al lumbricario; cantidad de litros de humus líquido que obteníamos; fracciones y proporciones en la cantidad de humus en tierra; y altura alcanzada por las plantas y la presencia del calendario en el tiempo de germinación), representación de gráficas de crecimiento, control de variables dependientes e independientes (tipo de humus y tipo se semillero) sobre todo durante la fase 4.

- Área de Lengua Castellana: texto instructivo y comprensión oral para la fabricación del lumbricario; y la realización de escritos a expertos en humus de lombriz a través del correo electrónico para preguntar dudas que nos iban surgiendo. Algunos ejemplos por fases de trabajo: aspectos formales de corrección, cohesión, coherencia y adecuación de los textos (fase 1), expresión oral para atender a la siguiente cuestión: ¿Cómo solicitar ayuda frente a para nuestra investigación a los expertos? (fase 2), y redacción de las conclusiones del proceso de investigación (fase 4).

- Otras áreas: trabajo colaborativo y en equipo, respeto por las ideas de los compañeros/as, trabajo autónomo, aprender a prender, uso adecuado y respeto de los materiales, expresión artística, creatividad, etc. Principalmente en la fase 3 a partir de la elaboración de carteles y diseño del invernadero y la zona para llevar a cabo el trabajo. Y en la fase 2 fue necesario trabajar con normas para la prevención de riesgos laborales, así como una actitud positiva hacia el trabajo en equipo y el respeto de las ideas de los compañeros/as independientemente de la raza, sexo o nivel académico. 
Tabla 6

Aprendizajes por fases de trabajo

Área de conocimiento

Fases

Ciencias Naturales

\begin{tabular}{|c|c|c|c|c|c|c|c|}
\hline \multirow{2}{*}{\multicolumn{2}{|c|}{ Fases }} & \multicolumn{3}{|c|}{ Ciencias Naturales } & \multirow{2}{*}{ Matemáticas } & \multirow{2}{*}{ Lengua } & \multirow{2}{*}{$\begin{array}{l}\text { Otras } \\
\text { áreas }\end{array}$} \\
\hline & & CC & $\mathrm{HC}$ & $\mathrm{AC}$ & & & \\
\hline \multicolumn{2}{|c|}{ Fase 1: Formulación del Problema } & $\mathrm{X}$ & $\mathrm{X}$ & $\mathrm{X}$ & & $\mathrm{X}$ & \\
\hline \multicolumn{2}{|c|}{ Fase 2: Planificación } & $\mathrm{X}$ & $\mathrm{X}$ & $\mathrm{X}$ & $\mathrm{X}$ & $\mathrm{X}$ & $\mathrm{X}$ \\
\hline \multirow{3}{*}{$\begin{array}{l}\text { Fase 3: } \\
\text { Experimentación }\end{array}$} & Subfase 3.1 & $\mathrm{X}$ & $\mathrm{X}$ & $\mathrm{X}$ & & & \\
\hline & Subfase 3.2 & $\mathrm{X}$ & $\mathrm{X}$ & $\mathrm{X}$ & $\mathrm{X}$ & & \\
\hline & Subfase 3.3 & $\mathrm{X}$ & $\mathrm{X}$ & $\mathrm{X}$ & $\mathrm{X}$ & & $\mathrm{X}$ \\
\hline \multicolumn{2}{|c|}{$\begin{array}{l}\text { Fase 4: Discusión de los resultadosy } \\
\text { conclusiones }\end{array}$} & $\mathrm{X}$ & $\mathrm{X}$ & $\mathrm{X}$ & $\mathrm{X}$ & $\mathrm{X}$ & \\
\hline
\end{tabular}

\section{Resultados y discusión}

Tras la experiencia educativa descrita a partir de la investigación realizada con las lombrices, se presentan algunos de los resultados más representativos atendiendo a las cinco categorías objeto de análisis a partir de la hoja de registro del docente (tabla 1). Para ello se contrasta la situación de partida o necesidad educativa detectada con la mejora alcanzada tras la experiencia, tal y como se recoge en la tabla 7 . Además, se ha complementado los resultados obtenidos a partir de la hoja de registro del docente, con otras anotaciones que configuran el diario del profesor (actividades y/o producciones del alumnado, participación, motivación e interés, etc.).

\section{Tabla 7}

Resultados de la experiencia educativa

\begin{tabular}{|c|c|c|c|}
\hline $\begin{array}{l}\text { Categorías } \\
\text { Observables }\end{array}$ & $\begin{array}{l}\text { Situación de Partida: } \\
\text { dificultades }\end{array}$ & $\begin{array}{l}\text { Momento o } \\
\text { Actividad }\end{array}$ & $\begin{array}{l}\text { Tras la experiencia: mejoras } \\
\text { observadas }\end{array}$ \\
\hline $\begin{array}{l}\text { I. Habilidades } \\
\text { cientificas }\end{array}$ & $\begin{array}{l}\text { - Gran parte del } \\
\text { alumnado no sabía } \\
\text { recoger, ni tomar } \\
\text { datos de forma } \\
\text { sistematizada } \\
\text { - No usaban } \\
\text { instrumentos de } \\
\text { medida o de recogida } \\
\text { de la información. } \\
\text { - La información } \\
\text { recogida era } \\
\text { principalmente en } \\
\text { base a la descripción } \\
\text { visual. } \\
\text { - La información } \\
\text { recogida no era } \\
\text { precisa, se basaban en } \\
\text { aspectos sumamente } \\
\text { subjetivos. }\end{array}$ & $\begin{array}{l}\text { 1. Actividades de } \\
\text { observación del } \\
\text { huerto para } \\
\text { determinar las } \\
\text { acciones que había } \\
\text { que realizar } \\
\text { diariamente para su } \\
\text { mantenimiento y } \\
\text { producción. } \\
\text { 2. Recogida de datos } \\
\text { a partir de la hoja de } \\
\text { registro: evolución } \\
\text { de las semillas } \\
\text { estaban plantadas en } \\
\text { el invernadero con y } \\
\text { sin humus de } \\
\text { lombriz. }\end{array}$ & $\begin{array}{l}\text { Las observaciones que realizan en } \\
\text { el huerto para su mantenimiento } \\
\text { son cada vez más precisas: } \\
\text { - Usan otros sentidos además } \\
\text { de la vista, como el tacto } \\
\text { (tocan la tierra para determinar } \\
\text { su textura, composición) e } \\
\text { incluso el olor. } \\
\text { - Mayor sistematización del } \\
\text { proceso de observación: } \\
\text { tenían más claro qué observar } \\
\text { (si funcionaba el riego, posible } \\
\text { presencia de invertebrados, } \\
\text { etc.), dónde y cómo recoger } \\
\text { los datos. } \\
\text { - Recogida de datos más } \\
\text { objetiva, basada en hechos } \\
\text { observables (aspecto de la } \\
\text { tierra y de la hierba, presencia } \\
\text { de animales, relación entre } \\
\text { ellos, etc.) y cuantitativas }\end{array}$ \\
\hline
\end{tabular}


(presencia de pulgón en cuentas plantas, añadir humus de lombriz a tal fracción de la plantación, que tanto por ciento de plantas se han secado...)

- Organización de las tareas más fluida y efectiva.

Disminuyendo el tiempo de asamblea necesario para ponerse en marcha

$\begin{array}{ll} & \begin{array}{l}\text { Gran parte del } \\ \text { alumnado iba al huerto } \\ \text { de visita y con el } \\ \text { objetivo principal de }\end{array} \\ \text { II. Actitudes } & \text { salir del aula. No } \\ \text { positivas hacia la } & \text { valoraban el huerto } \\ & \text { como un lugar de } \\ & \text { aprendizaje y por tanto } \\ & \text { de escaso interés. }\end{array}$

Gran parte del alumnado iba al huerto de visita y con el salir del aula. No valoraban el huerto aprendizaje y por tanto de escaso interés.
1. Durante las visitas semanales al huerto.

2. Durante el proceso de fabricación del humus.

3. Durante el proceso de registro de datos de su experiencia

Aumentó considerablemente el grado de implicación y cuidado hacia el huerto, valorándolo como algo suyo y un lugar donde podía aprender cosas interesantes sobre la ciencia desarrollando habilidades que en espacios como el aula no eran posibles. Por ejemplo, cuando el alumnado visitaba el huerto, lo hacía para detectar algún problema o mantenerlo: era capaz de percibir modificaciones y/o relaciones que existían en ese ecosistema y cómo poder intervenir en el mismo

\begin{tabular}{|c|c|c|c|}
\hline $\begin{array}{l}\text { II. Grado de } \\
\text { sensibilización } \\
\text { medioambiental }\end{array}$ & $\begin{array}{l}\text { El alumnado tiene una } \\
\text { visión del medio } \\
\text { sumativa y egocéntrica. } \\
\text { Reconoce que el huerto } \\
\text { ecológico no usa } \\
\text { compuestos químicos } \\
\text { sintéticos porque } \\
\text { relaciona este hecho } \\
\text { con el efecto positivo } \\
\text { para los nutrientes de } \\
\text { los alimentos, pero no } \\
\text { reconoce las ventajas } \\
\text { del huerto ecológico } \\
\text { para la preservación del } \\
\text { suelo, o posibles } \\
\text { alteraciones químicas } \\
\text { de los acuíferos. }\end{array}$ & $\begin{array}{l}\text { 1. Durante el proceso } \\
\text { de fabricación del } \\
\text { humus y cuidado } \\
\text { del lumbricario. }\end{array}$ & $\begin{array}{l}\text { Con esta práctica entendió que, } \\
\text { con el conocimiento del suelo y la } \\
\text { relación entre el ecosistema, se } \\
\text { puede y se debe intervenir para la } \\
\text { optimización de los recursos de } \\
\text { forma ecológica, obteniendo } \\
\text { resultados sin variar el equilibrio } \\
\text { del ecosistema ni las características } \\
\text { del producto cosechado. } \\
\text { Se cambió hábitos, relacionados } \\
\text { con el consumo, comprando } \\
\text { alimentos que fueran más } \\
\text { sostenibles. }\end{array}$ \\
\hline $\begin{array}{l}\text { III. Grado de } \\
\text { adquisición } \\
\text { de Hábitos } \\
\text { saludables }\end{array}$ & $\begin{array}{l}\text { Gran parte del } \\
\text { alumnado, o más de la } \\
\text { mitad, reconocen no } \\
\text { tomar verduras de } \\
\text { forma habitual en su } \\
\text { dieta e incluso ni } \\
\text { probarla. }\end{array}$ & $\begin{array}{l}\text { 1. Durante la recolecta } \\
\text { de la siembra y } \\
\text { posterior } \\
\text { elaboración y } \\
\text { cocinado de los } \\
\text { alimentos } \\
\text { recolectados. }\end{array}$ & $\begin{array}{l}\text { Más de la mitad del alumnado } \\
\text { introdujo en su dieta semanal } \\
\text { algún tipo de verdura y casi todos } \\
\text { la probaban al menos. En la hora } \\
\text { del recreo se observaba también } \\
\text { que había más niños/as que traían } \\
\text { frutas y otras meriendas más } \\
\text { saludables. }\end{array}$ \\
\hline $\begin{array}{l}V . \\
\text { Comportamiento } \\
\text { y uso de los }\end{array}$ & $\begin{array}{l}\text { Parte del alumnado no } \\
\text { tenía un } \\
\text { comportamiento } \\
\text { adecuado para trabajar }\end{array}$ & $\begin{array}{l}\text { 1. Durante las } \\
\text { actividades } \\
\text { rutinarias de }\end{array}$ & $\begin{array}{l}\text { El alumnado se organizaba de } \\
\text { forma autónoma para las tareas de } \\
\text { mantenimiento del huerto; } \\
\text { seleccionaba con mayor criterio la }\end{array}$ \\
\hline
\end{tabular}




\begin{tabular}{|c|c|c|c|}
\hline $\begin{array}{l}\text { materiales e } \\
\text { instrumentos }\end{array}$ & $\begin{array}{l}\text { en el huerto: no } \\
\text { tomaba medidas de } \\
\text { prevención mínimas } \\
\text { con los instrumentos y } \\
\text { herramientas de } \\
\text { labranza; no solían } \\
\text { ordenar y mantener las } \\
\text { herramientas en su } \\
\text { lugar correspondiente; } \\
\text { y no trabajan de forma } \\
\text { organizada ni } \\
\text { cooperativa } \\
\text { apareciendo muchas } \\
\text { veces momentos de } \\
\text { conflicto en el aula. }\end{array}$ & $\begin{array}{l}\text { mantenimiento } \\
\text { del huerto }\end{array}$ & $\begin{array}{l}\text { herramienta a usar; tenían en } \\
\text { consideración la distancia de } \\
\text { seguridad que debían guardar con } \\
\text { su compañero/a; y la limpiaban y } \\
\text { la guardaban en su lugar. } \\
\text { Igualmente, disminuyeron muchos } \\
\text { de los comportamientos } \\
\text { disruptivos rutinarios del aula y } \\
\text { otro tipo de incidentes } \\
\text { relacionados con la falta de } \\
\text { actitudes de respecto y } \\
\text { colaboración. }\end{array}$ \\
\hline
\end{tabular}

Como se recoge en la tabla 7, las distintas categorías objeto de análisis han ido mejorando en el alumnado, en particular, sus habilidades y conocimientos científicos. No menos relevante son las actitudes positivas hacia la ciencia, a partir del interés mostrado a lo largo de todo el proyecto por mejorar la producción del huerto a partir de su mantenimiento. Similares resultados encontraron Klemmer et al. (2005) trabajando la huerta como recurso para la enseñanza de las ciencias con niños/as de educación primaria. Gran parte de estas mejoras han sido gracias al fomento del trabajo en equipo y colaborativo, la mejora de las relaciones y comportamientos entre los estudiantes (Robinson \& Zajicek, 2005; Ruiz-Gallardo et al., 2013), así como el respeto por los materiales y herramientas usadas en el huerto. Resaltar que durante toda la experiencia no ha habido problemas de conductas entre los estudiantes, se han respetado las pautas de trabajo, creándose una rutina de trabajo autónomo. Señalar también el impacto positivo en la promoción de hábitos saludables, a partir del consumo e introducción en la dieta alimenticia de frutas y verduras, en los estudiantes. Este es un aspecto que los estudios de Morgan et al. (2010) y Ratcliffe et al. (2011) también se lo atribuyen al trabajar con los huertos escolares de forma cotidiana e integrada en los programas curriculares. Igualmente, desde que se viene usando este tipo de experiencias, el centro ha observado una disminución del absentismo y mejora de las competencias de las distintas áreas de conocimiento, de la misma forma lo expresan otras propuestas educativas de aprendizaje basado en proyectos con alumnado absentista y comportamientos disruptivos como el Daza et al. (2020).

Estos resultados generales se complementan con los siguientes (figuras 5-8) que presentan en detalle algunos resultados específicos de determinados descriptores de cada categoría. Los resultados que representan los gráficos son porcentuales, donde nos permite contrastar los resultados iniciales a la experiencia o pre, con los obtenidos al final de la experiencia o post.

Como podemos comprobar en la figura 5 , es notable los cambios que se producen en relación con la mejora de las Habilidades científicas en cuanto a la toma de datos y medidas. En la situación de partida (pre) vemos como como el $80 \%$ del alumnado no sabían medir datos y solo un $20 \%$ lo hacían de forma poco precisa. Tras la experiencia el 70\% del alumnado comienza a medir y cuantificar de forma precisa.

\section{Figura 5}

Habilidades cientificas (medición y toma de datos)




En relación a la categoría Actitudes positivas hacia la ciencia (figura 6) uno de los aspectos que se valoraban en los descriptores era el respeto de las normas de seguridad en el huerto con el manejo de las herramientas y su cuidado. Al comienzo de la experiencia (pre) solo un $20 \%$ del alumnado mostraba un comportamiento adecuado, y tras la experiencia el $90 \%$ de la clase usaba y manejaba de forma segura las herramientas del huerto.

\section{Figura 6}

Actitudes positivas hacia las ciencias (respeto de las normas de seguridad)

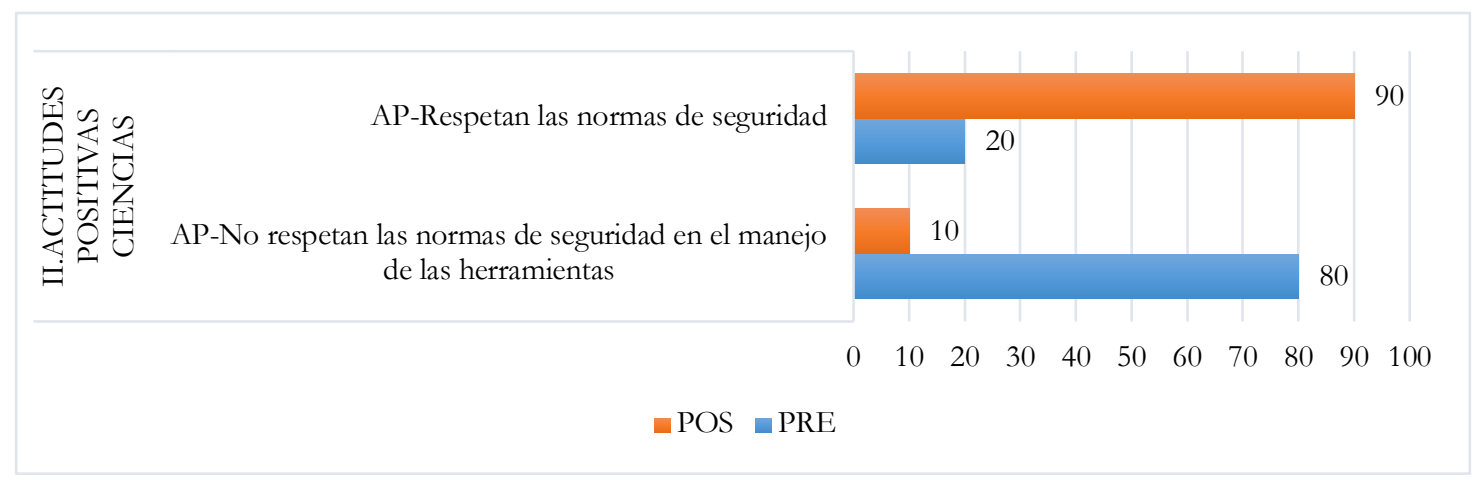

La figura 7, se corresponde con la categoría Sensibilización ambiental y el descriptor actuaciones responsables $y$ sostenibles hacia el medioambiente. Como se observa antes de la experiencia el $70 \%$ del alumnado no era consciente de los problemas medioambientales y su relación con los hábitos alimenticios y de consumo responsable. Tras la experiencia no solo el $80 \%$ del alumnado toma conciencia de esta problemática, sino que un $20 \%$ comienza a integrar en su día a día acciones respetuosas y sostenibles.

\section{Figura 7}

Actitudes positivas hacia las ciencias (respeto de las normas de seguridad)

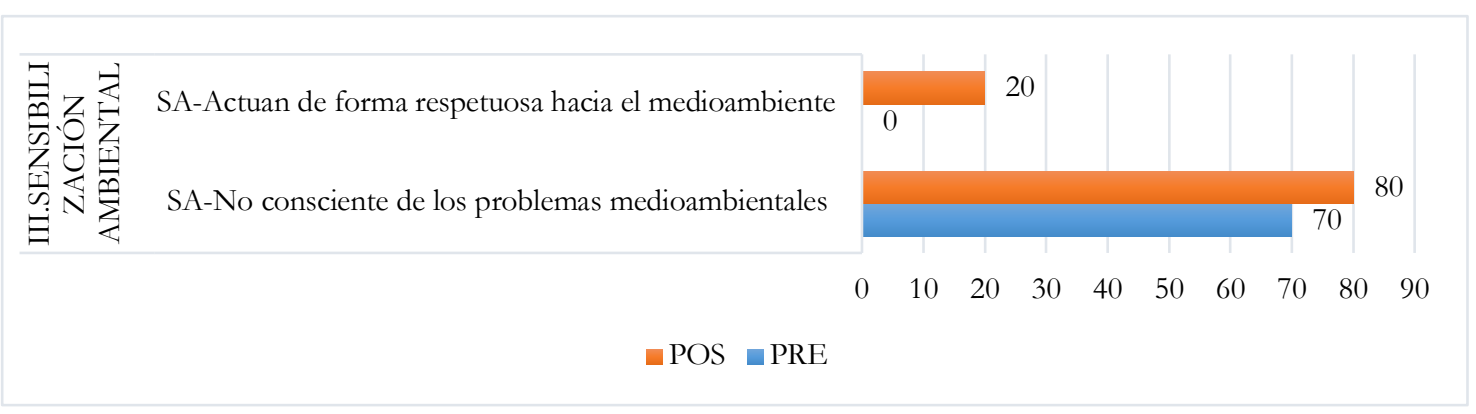

En la categoría Adquisición de hábitos saludables y el descriptor dieta saludable (figura 8), se observa el impacto positivo que tiene esta experiencia en la integración de su dieta de frutas y verduras. El 50\% del alumnado declaraba no probar muchas frutas y verduras comunes de nuestra dieta mediterránea, tras la experiencia, hemos conseguido que un $65 \%$ de la clase comience a incluir estos alimentos en su dieta semanal. 


\section{Figura 8}

Adquisición de bábitos saludables (mejora de la dieta)

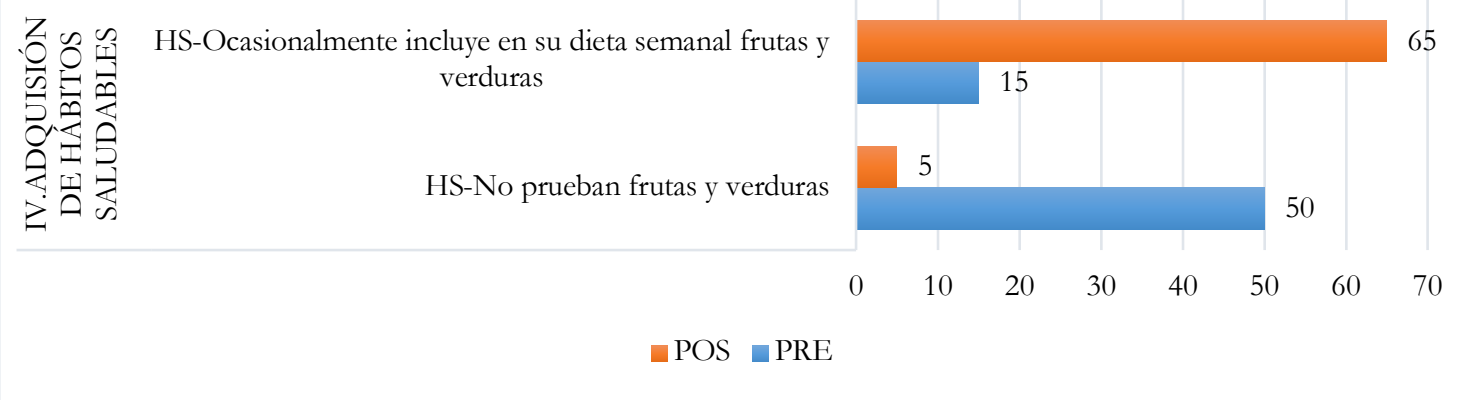

Por último, recordar que, aunque los conocimientos científicos no han sido objeto directo de análisis en este trabajo (para ello se han usado otros instrumentos específicos) a partir de la mejora de las categorías observables estudiadas podemos advertir de forma indirecta un mayor conocimiento y comprensión de conceptos asociados al campo de la biología (ciclo de vida de las lombrices), de la geología (formación del suelo y mantenimiento) y de ecología (visión sistémica del huerto).

\section{Conclusiones}

Con la exposición de esta experiencia didáctica a partir del uso del huerto escolar como recurso educativo, deseamos alentar a otros docentes a que realicen más propuestas educativas fuera del aula para promocionar un aprendizaje contextualizado con su alumnado. No obstante, somos conscientes de los obstáculos y dificultades que tienen los maestros de primaria para realizar este tipo de experiencias innovadoras, derivadas principalmente de la falta de apoyo del centro y de recursos, así como de formación docente permanente (Daza et al., 2020) sucediendo igualmente en otros contextos internacionales como señalan Dring et al. (2020). A pesar de ello, este momento de crisis sanitaria, que nos ha "empujado" a salir del aula hacia espacios abiertos para disminuir la probabilidad de contagio por COVID-19, puede ser un buen momento para explorar este recurso educativo que es ilimitado en affordances. Son inagotables las posibilidades didácticas del huerto escolar para enseñar cualquier conocimiento disciplinar, mejorar actitudes, comportamientos, promocionar hábitos saludables (Eugenio-Gozalbo et al., 2019; EugenioGozalbo et al., 2020), además de fomentar actitudes sociocríticas para el decrecimiento (Rodríguez-Marín, et al., 2015) tan necesarias para combatir el colapso medioambiental de los sistemas socioeconómicos. En este sentido, como ya se expuso, no son todavía muchos los colegios que disponen de huertos escolares en Sevilla, un 29\% de 128 escuelas estudiadas (privadas, concertadas y públicas) disponen de estos espacios (Vílchez y Escobar, 2014). Un incipiente incremento o tendencia superior parece que se registra en los últimos años en la provincia sevillana: 39\% de huertas escolares en 63 centros educativos (Ceballos, 2017).

Por otro lado, el haber desarrollado esta experiencia desde el huerto escolar y registrarla en nuestro diario docente, nos ha ayudado a reflexionar sobre nuestra praxis para así mejorar futuras intervenciones didácticas, al igual que expresan otros trabajos previos sobre la temática (Eugenio-Gonzalvo et al., 2019).

Finalmente, recordar que este trabajo y sus resultados corresponden a un grupo de clase con un número limitado de estudiantes, por lo que debe ser entendido como una primera aproximación al fenómeno estudiado. Usar otros instrumentos de recogida y análisis para medir el impacto educativo de esta experiencia pueden ayudarnos a obtener resultados más fiables y extrapolables a otros escenarios parecidos.

\section{Referencias}

Abril-López, D., Morón-Monge, H., Morón-Monge, M. D. C., \& López Carrillo, M. D. (2021). The Learning to Learn Competence in Early Childhood Preservice Teachers: An Outdoor and e/m-Learning Experience in the Museum. Future Internet, 13(2), 25.

Aguilera, D. (2018). La salida de campo como recurso didáctico para enseñar ciencias. Una revisión sistemática. Revista Eureka sobre Enseñanza y Divulgación de las Ciencias, 15(3) 3103-3103. 10.25267/Rev_Eureka_ensen_divulg_cienc.2018.v15.i3.3103 
Amórtegui, E., Mayoral, O. y Gavidia., V. (2017). Aportaciones de las Prácticas de Campo en la formación del profesorado de Biología: un problema de investigación y una revisión documental. Didáctica de las ciencias experimentales y sociales, (32), 153-170. http:// hdl.handle.net/10550/63122

Bisquerra, R. (2009). Metodología de la Investigación Educativa (2nd ed.). La Muralla.

Carballido, JL. (2020). ¿Cómo trabajar la ciencia en el huerto escolar? [Producción videográfica]. Coordinadora del proyecto Daza-Navarro, P. y col (Departamentos: Biología Celular y Didáctica de las Ciencias Experimentales). Producido por el SAV, Secretaría técnica de Recursos y Audiovisuales de la Universidad de Sevilla. https://tv.us.es/como-trabajar-la-ciencia-en-el-huerto-escolar/

Ceballos, M. (2017). Aprovechamiento didáctico de los huertos escolares en centros de Sevilla. Enseñanźa de las ciencias, (Extra), 0787-792. https://www.raco.cat/index.php/Ensenanza/article/view/334757

Contreras, S.A. (2016). Pensamiento Pedagógico en la Enseñanza de las Ciencias: Análisis de las Creencias Curriculares y sus Implicancias para la Formación de Profesores de Enseñanza Media. Formación Universitaria, 9(1), 15-24. http://dx.doi.org/10.4067/S0718-50062016000100003

COSCE (2011). Informe ENCIENDE. Enseñanza de las Ciencias en la Didáctica Escolar para edades tempranas en España. Ministerio de Ciencias e Innovación. COSCE. Recuperado de http:// www.cosce.org/pdf/Informe_ENCIENDE.pdf

Costillo, E., Borrachero, A. B., Villalobos, A. M., Mellado, V., y Sánchez, J. (2014). Utilización de la modelización para trabajar salidas al medio natural en profesores en formación de educación secundaria. Biografía Escritos sobre la biología y su enseñanza, 7(13), 165-175. 10.17227/20271034.vol.7num.13bio-grafia165.175

Daza, M.., Morón-Monge, H., y Daza-Navarro, P. (2020). El trabajo por proyectos en Educación Secundaria Obligatoria. Una experiencia desde el Departamento de Inglés: My Experience Abroad. Revista Andina de Educación, 3(2), 32-40. https://doi.org/10.32719/26312816.2020.2.3.5

Denzin, N.K. \& Lincoln, Y.S. (2012). Strategies of Qualitative Inquiry (4th ed.). Sage.

Dillon, J., Rickinson, M., Teamey, K., Morris, M., Choi, M. Y., Sanders, D. \& Benefield, P. (2006). The value of outdoor learning: evidence from research in the UK and elsewhere. School science review, 87(320), 107.

Dring, C. C., Lee, S. Y. \& Rideout, C. A. (2020). Public school teachers' perceptions of what promotes or hinders their use of outdoor learning spaces. Learning Environments Research, 23(3), 369-378.

Eugenio-Gozalbo, M., Pérez-López, R. y Tójar-Hurtado, J. C. (2020). Identifying key issues for university practitioners of garden-based learning in Spain. The Journal of Environmental Education, 51, 246-255. https://doi.org/10.1080/00958964.2019.1687407

Eugenio-Gozalbo, M., Ramos, G. y Vallés, C. (2019). Huertos universitarios: dimensiones de aprendizaje percibidas por los futuros maestros, Enseñanza de las ciencias, 37(3), 111-127. https://doi.org/10.5565/rev/ensciencias.2657

Fernández-Arroyo, J., Puig, M. y Rodríguez-Marín, F. (2013). El uso del huerto escolar en la formación del profesorado de magisterio a través de la práctica educativa. In M. A., Ballesterios-Moscopios y F., Ries (Eds), International Conference Re-conceptualizing the professional identity of the European teacher. Sharing Experiences (pp. 699-716). Copiarte. http://hdl.handle.net/11441/56705

Ferrés Gurt, C., Marbà Tallada, A. y Sanmartí Puig, N. (2014). Trabajos de indagación de los alumnos: instrumentos de evaluación e identificación de dificultades. Revista Eureka sobre Enseñanza y Divulgación de las Ciencias, 12(1), 22-37. http://hdl.handle.net/10498/16922

Franco Mariscal, A. J., Blanco López, Á. y España Ramos, E. (2017). Diseño de actividades para el desarrollo de competencias científicas. Utilización del marco de PISA en un contexto relacionado con la salud. Revista Eureka sobre Enseñanza y Divulgación de las Ciencias, 14(1), 38-53.

Fuertes Camacho, M. T. (2011). La observación de las prácticas educativas como elemento de evaluación y de mejora de la calidad en la formación inicial y continua del profesorado. Revista de docencia universitaria, 9(3), 237-258

Gelter, H. (2000). Friluftsliv: The Scandinavian philosophy of outdoor life. Canadian Journal of Environmental Education (CJEE), 5(1), 77-92.

Gibson, J.J. (1979). The ecological approach to visual perception. Houghton-Mifflin.

Godoy, A. V., Segrra, C. I. y Di Mauro, M. F. (2014). Una experiencia de formación docente en el área de Ciencias Naturales basada en la indagación escolar. Revista Eureka sobre Enseñanza y Divulgación de las Ciencias, 11(3), 381-397.

Harris, I. (1999). Outdoor Education in Secondary Schools: What Future? Horizons, 4, 5-8.

Hodson, D. (2014). Becoming part of the solution: Learning about activism, learning through activism, learning from activism. In J. L. Bencze y S. Alsop (Eds.), Activist science and technology education (pp. 6798). Springer. 
Klemmer, C. D., Waliczek, T. M. \& Zajicek, J. M. (2005). Development of a science achievement evaluation instrument for a school garden program. HortTechnology, 15(3), 433-438. https://doi.org/10.21273/HORTTECH.15.3.0433

Ley Orgánica 8/2013, 9 diciembre, para la mejora de la calidad educativa (LOMCE); Ministerio de la Presidencia (Boletín Oficial del Estado, 295): Madrid, Spain, 2013; pp. 97858-97921; Disponible: https://www.boe.es/boe/ dias/2013/12/10/pdfs/BOE-A-2013-12886.pdf

Maldonado, S., Ospino, L., Martínez, J., Salguedo, G., Salcedo, L. y Ospino, D. (2018). Implementación de una huerta escolar como herramienta estratégica para fomentar la investigación. Cultura. Educación y Sociedad, 9(3), 335-342. http://dx.doi.org/10.17981/cultedusoc.9.3.2018.38

Monsalves, A. Y. y Pérez, E. M., (2012). El diario pedagógico como herramienta para la investigación. Itinerario educativo, 26(60), 117-128.

Morales, A. J., Caurín, C., Sendra, C. y Parra, M. (2014). Aprendiendo a plantear problemas en el medio. Análisis de una experiencia con estudiantes del Máster de Investigación en Didácticas específicas. Didáctica de las Ciencias Experimentales y Sociales, (28), 65-81. https://doi.org/10.7203/DCES.28.3848.

Morgan, P. J., Warren, J. M., Lubans, D. R., Saunders, K. L., Quick, G. I. \& Collins, C. E. (2010). The impact of nutrition education with and without a school garden on knowledge, vegetable intake and preferences and quality of school life among primary-school students. Public health nutrition, 13(11), 1931-1940. https://doi.org/10.1017/s1368980010000959

Morón-Monge, H., Morón-Monge, M. D. C., Abril-López, D., \& Daza Navarro, M. P. (2020). An Approach to Prospective Primary School Teachers' Concept of Environment and Biodiversity through their Design of Educational Itineraries: Validation of an Evaluation Rubric. Sustainability, 12(14), 5553.

Nicolás, A. M. B., Soler, A. H. y Doménech, J. C. (2014). Las competencias básicas a través del huerto escolar: una propuesta de proyecto de innovación. Investigación e innovación en formación del profesorado, 173182.

OCDE (2005). Informe PIS A 2003. Aprender para el mundo de mañana. Santillana.

Orden 17 marzo 2015, por la que se Desarrolla el Currículo Correspondiente a la Educación Primaria en Andalucía; Consejería de Educación de la Junta de Andalucía (Boletín Oficial de la Junta de Andalucía, 60): Sevilla, España, 2015; pp. 9-696; Disponible: https://www.juntadeandalucia.es/boja/2015/60/1

Pedrinaci, E., Caamaño A., Cañal P. y de Pro A., (2012). 11 ideas clave: el desarrollo de la competencia científica. Grao.

Porlán, R. y Martín, J., (2000). El diario del Profesor. Un recurso para la investigación en el aula. Diada Editora.

Ratcliffe, M. M., Merrigan, K. A., Rogers, B. L. \& Goldberg, J. P. (2011). The effects of school garden experiences on middle school-aged students' knowledge, attitudes, and behaviors associated with vegetable consumption. Health promotion practice, 12(1), 36-43. https://doi.org/10.1177/1524839909349182

Rekalde, I., Vizcarra, M. T. y Macazaga, A. M. (2014). La observación como estrategia de investigación para construir contextos de aprendizaje y fomentar procesos participativos. Educación XX1, 17(1), 201-220.

Robinson, C. W. \& Zajicek, J. M. (2005). Growing minds: The effects of a one-year school garden program on six constructs of life skills of elementary school children. HortTechnology, 15(3), 453-457. https://doi.org/10.21273/HORTTECH.15.3.0453

Rocard, M., Csermely, P., Jorde, D., Lenzen, D., Walberg-Henriksson, H., \& Hemmo, V (2007). Science Education NOW: A renewed Pedagogy for the Future of Europe. European Commission.

Rodríguez-Marín, F., Fernández-Arroyo, J. y García Díaz, J. E. (2015). El huerto escolar ecológico como herramienta para la educación en y para el decrecimiento. Investigación en la Escuela, (86), 35-48. https://doi.org/10.12795/IE.2015.i86.03

Ruiz-Gallardo, J., Verde, A. y Valdes, A. (2013). Garden-based learning: an experience with "at risk" secondary education students. The Journal of Environmental Education, 44, 252-270. https://doi.org/10.1080/00958964.2013.786669

Sadler, T. D. (2004). Moral and ethical dimensions of socioscientific decision-making as integral components of scientific literacy. Science Education, 13, 39-48.

Sáez Bondía, M. J., Lucha López, P., Claver Giménez, A. M., Arasanz Broto, Á. y Iráizoz Esteban, R. (2017). Del dicho al hecho en una propuesta sobre ecosistemas contextualizada en el huerto escolar. Apice, 1(2), 47-57.

Sanz, J., Zuazagoitia, D., Pérez, M. Lizaso, E. (2021). Análisis de la potencialidad de los espacios al aire libre para trabajar la competencia científica a través del alumnado en formación. En Ápice y Universidad de Córdoba (Eds), 29 Encuentros Didáctica de las Ciencias experimentales (pp. 1427-1434). Córdoba: Universidad de Córdoba. 
Tal, R. T. \& Morag, O. (2009). Reflective practice as a means for preparing to teach outdoors in ecological garden. Journal of Science Teacher education, 20(39), 245-262. https://doi.org/10.1007/s10972-009-9131-1

Vílchez, J. E. y Escobar, T. (2014). Uso de laboratorio, huerto escolar y visitas a centros de naturaleza en Primaria: Percepción de los futuros maestros durante sus prácticas docentes. Revista Electrónica de Enseñanza de las Ciencias, 13(2), 222-241.

Vílchez, J. E., Escobar, T. y Ceballos, M. (2012). Prioridad y asociaciones en el uso de materiales para la enseñanza de las ciencias en centros escolares de Primaria. En J.M., Domínguez (Ed), XXV Encuentro de Didáctica de las Ciencias Experimentales (pp. 1321-1328). Santiago de Compostela: Universidad de Santiago de Compostela.

Waite, S. (2011). Teaching and learning outside the classroom: Personal values, alternative pedagogies and standards. Education 3-13, 39(1), 65-82. https://doi.org/10.1080/03004270903206141

Zamalloa, T., Sanz, J., Maguregi, G., Echevarría, I., Fernández, L. (2014) Acercar la geodiversidad a través de las salidas de campo en la ESO. Una investigación con el profesorado de ciencias de Bizkaia. Enseñanza de las ciencias: revista de investigación y experiencias didácticas, 32(3), 443-467. $10.5565 / \mathrm{rev} /$ ensciencias. 1282

Zambrano-Quintero, Y., Rocha-Roja, C., Flórez-Vanegas, G., Nieto-Montaño, L., Jiménez-Jiménez, J. y Núñez-Samnández, L. (2018). La huerta escolar como estrategia pedagógica para fortalecer el $\begin{array}{lllll}\text { aprendizaje. } \quad \text { Cultura. } & \text { Educación }\end{array}$ http://dx.doi.org/10.17981/cultedusoc.9.3.2018.53

Zeidler, D. L., Sadler T. D., Simmons. M. L., y Howes, E.V. (2005). Beyond STS: A research-based framework for socioscientific issues education. Science Education, 89(3), 357-377. https://doi.org/10.1002/sce.20048 